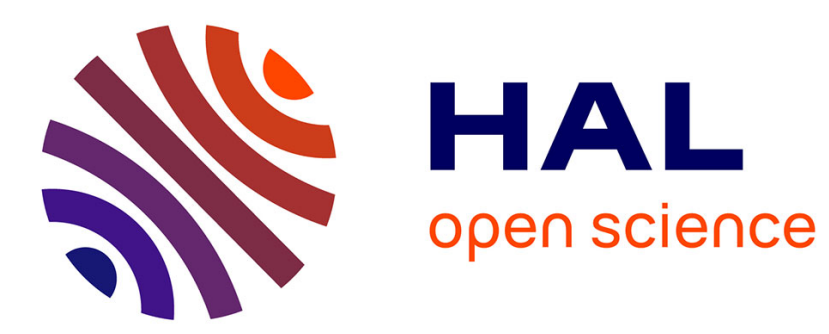

\title{
Perception gustative et couverture des besoins énergétiques chez les primates
}

Bruno Simmen, Patrick Pasquet, Claude Marcel Hladik

\section{To cite this version:}

Bruno Simmen, Patrick Pasquet, Claude Marcel Hladik. Perception gustative et couverture des besoins énergétiques chez les primates. 2013. hal-00819775v2

\section{HAL Id: hal-00819775 \\ https://hal.science/hal-00819775v2}

Preprint submitted on 13 Nov 2013

HAL is a multi-disciplinary open access archive for the deposit and dissemination of scientific research documents, whether they are published or not. The documents may come from teaching and research institutions in France or abroad, or from public or private research centers.
L'archive ouverte pluridisciplinaire HAL, est destinée au dépôt et à la diffusion de documents scientifiques de niveau recherche, publiés ou non, émanant des établissements d'enseignement et de recherche français ou étrangers, des laboratoires publics ou privés. 


\title{
Perception gustative et couverture des besoins énergétiques chez les primates
}

\author{
Gustatory perception and energy expenditure in primates
}

\author{
Bruno Simmen, Patrick Pasquet et Claude Marcel Hladik
}

Eco-anthropologie et Ethnobiologie, UMR 7206, CNRS, Université Paris-7 et Muséum national d'Histoire naturelle. Laboratoire d'Ecologie Générale, 1 avenue du Petit Château. F 91800 Brunoy. e-mail :simmen@mnhn.fr

\section{Résumé}

La perception gustative permet d'orienter les choix alimentaires des primates dans les différentes niches écologiques où ils ont évolué. Les possibilités de choix sont cependant limitées par le poids corporel des différentes espèces, selon des relations allométriques dont nous présentons les caractéristiques chez les lémuriens et les primates platyrrhiniens et catarrhiniens par rapport aux autres ordres de mammifères. Le métabolisme globalement économe en énergie des primates, déterminé avec une précision suffisante chez plusieurs espèces incluant Homo sapiens, ainsi que les déterminants phylogénétiques et allométriques de la perception gustative, permettent de préciser l'origine des variations interspécifiques de la stratégie alimentaire.

\section{Mots clés}

Relations allométriques, perception du fructose, inertie phylogénétique, dépense énergétique, Lemuridae, Callitrichidae, Homininae

\section{Summary}

Food choices of primate species are driven by gustatory perceptions in the various ecological niches where each species has evolved. However, food choices are constrained by species' body mass, according to allometric relationships that are presented and discussed here. From the scaling of primate energetics, thrifty energy strategies seem to characterize Primates compared with other mammalian orders. In this paper, we analyze primate feeding strategies according to species variation in the field metabolic rate, as determined with an accurate method in several lemurs, platyrrhine and catarrhine species (including Homo sapiens). In this analysis, we take into account the phylogenetic and allometric effects on taste perception.

\section{Keywords}

Allometric relationships, perception of fructose, phylogenetic inertia, energy expenditure, Lemuridae, Callitrichidae, Homininae

\section{Introduction}

Les dépenses énergétiques des primates et les bilans qui en résultent ont récemment fait l'objet d'une revue présentée en ligne [1] avec des techniques de mesure qui apportent une précision suffisante pour mettre en perspective les adaptations par rapport aux stratégies alimentaires des différentes espèces. De telles mesures de la dépense énergétique en conditions naturelles pourraient permettre de préciser les variations des stratégies alimentaires au cours de l'évolution des homininés.

Si nous situons l'homme moderne par rapport aux autres mammifères, la relation entre ses besoins métaboliques de base et sa masse corporelle est bien connue depuis la publication du célèbre ouvrage de Max Kleiber en 1961, qui fait encore référence [2]. Cette relation dite allométrique (c'est-à-dire, associant 
une variable à une dimension corporelle), relie la dépense énergétique d'un individu au repos à jeun avec son poids. Elle varie de façon exponentielle et est figurée généralement sous la forme d'une droite lorsque les paramètres sont exprimés en logarithmes. Chez les mammifères, une relation allométrique s'applique également à la dépense énergétique journalière totale, et les variations mises en évidence entre espèces de même taille reflètent leurs différentes adaptations énergétiques globales à l'environnement [3]. Comment se situent les primates par rapport à cette relation de la dépense énergétique à la masse corporelle reste une question à explorer.

D'autres relations allométriques ont été recherchées à propos de la perception gustative, une modalité sensorielle essentielle à la couverture des besoins nutritionnels. Les capacités sensorielles d'une espèce peuvent-elles ainsi dépendre de sa dimension corporelle ? Cette question a été envisagée à propos de la perception de substances sucrées qui correspondent généralement à des aliments à forts apports énergétiques. De fait, la perception gustative dont nous présenterons les particularités à travers les seuils de sensibilité des primates actuels, a nécessairement co-évolué avec les stratégies des espèces suivant leur adaptation aux transformations des milieux [4]. L'utilisation de nouvelles ressources alimentaires repose en effet sur une combinaison de ces adaptations psychophysiologiques et comportementales visant à réguler la dépense énergétique totale. Dans la présente note, nous envisageons les origines des interactions sensorielles et métaboliques concernant l'Homme par une approche comparative de la sensibilité gustative et de la dépense énergétique totale en fonction de la masse corporelle des différentes espèces.

\section{Matériels et méthodes}

\section{Seuils de perception gustative}

Les seuils de perception gustative sont mesurés avec différentes méthodes chez les primates non- humains et chez l'Homme [5]. Chez l'Homme, la perception de produits purs dilués dans l'eau est déterminée en présentant une série de solutions en concentrations croissantes jusqu'à l'identification de la saveur (seuil de reconnaissance). On utilise généralement un éventail de substances sapides (des sucres, des acides organiques, des tannins...) permettant d'opérer en simple aveugle. Chez les primates non-humains, on utilise également une série de concentrations pour la substance à tester, en les présentant dans un ordre aléatoire à chaque test. Chacune des concentrations est présentée simultanément à de l'eau pure (le «two-bottle test ») au cours de tests de durée limitée sur des animaux à jeun. Lorsque la différence de consommation entre les deux fluides est significative, on considère que l'animal perçoit le goût de la solution. À partir d'une série de tests effectués sur plusieurs semaines, on détermine la concentration la plus faible du produit pour laquelle le sujet exprime encore une différence de consommation. Les seuils gustatifs ainsi mesurés avec une méthode comportementale s'avèrent semblables ou très proches de ceux déterminés par les enregistrements du potentiel évoqué sur le nerf de la gustation de différentes espèces de primates non-humains [5].

\section{Dépense énergétique}

La dépense énergétique totale par unité de temps d'un animal en conditions naturelles ou d'un humain est mesurée à l'aide de la méthode de l'eau doublement marquée [1]. Cette méthode exige, chez l'animal, la capture/recapture des individus afin de suivre les variations de la composition sanguine liées au métabolisme [6]. Après injection (ou absorption orale contrôlée chez l'homme) d'une quantité définie d'eau isotopique $\left({ }^{2} \mathrm{H}\right.$ et $\left.{ }^{18} \mathrm{O}\right)$, on mesure la production de dioxyde de carbone en fonction de l'élimination progressive et différentielle des isotopes stables de l'hydrogène et de l'oxygène au cours du temps. La production de $\mathrm{CO}_{2}$ est calculée par la différence de concentration entre les deux isotopes dans des prises de sang ou collecte d'urine (chez l'homme) successives : l'oxygène lié au dioxyde de carbone et à l'eau résultant des réactions métaboliques est éliminé deux fois plus rapidement que l'hydrogène lié uniquement à l'eau. Les concentrations isotopiques dans les différents échantillons sont mesurées avec un spectromètre de masse et une table de conversion permet de calculer la dépense énergétique réalisée entre les prélèvements. Il s'agit de la seule méthode permettant une mesure de grande précision de la dépense énergétique en conditions naturelles. 


\section{Résultats}

\section{Allométrie et seuil de perception du saccharose}

Les résultats présentés ont été compilés à partir des données obtenues et synthétisés par Glaser [7] ainsi qu'au cours de plusieurs études réalisées par les auteurs et leurs collaborateurs $[4,8]$. En regroupant l'ensemble des résultats concernant la perception des solutions de saccharose, on met en évidence une corrélation significative entre le format corporel et le seuil de perception gustative de cet hydrate de carbone. Cette relation est représentée par une droite après transformation logarithmique des valeurs mesurées (Fig. 1). En dépit d'une grande variabilité interspécifique qui se rapporte essentiellement aux différents régimes alimentaires, la plus grande acuité gustative vis-à-vis du saccharose (c'est-à-dire un plus faible seuil de perception mesuré) s'observe pour des espèces de plus grande masse corporelle. L'espèce humaine, qui se situe parmi les plus grands primates, est de fait l'une des plus sensibles au goût sucré du saccharose, avec une acuité gustative qui varie sensiblement dans différentes populations [9].

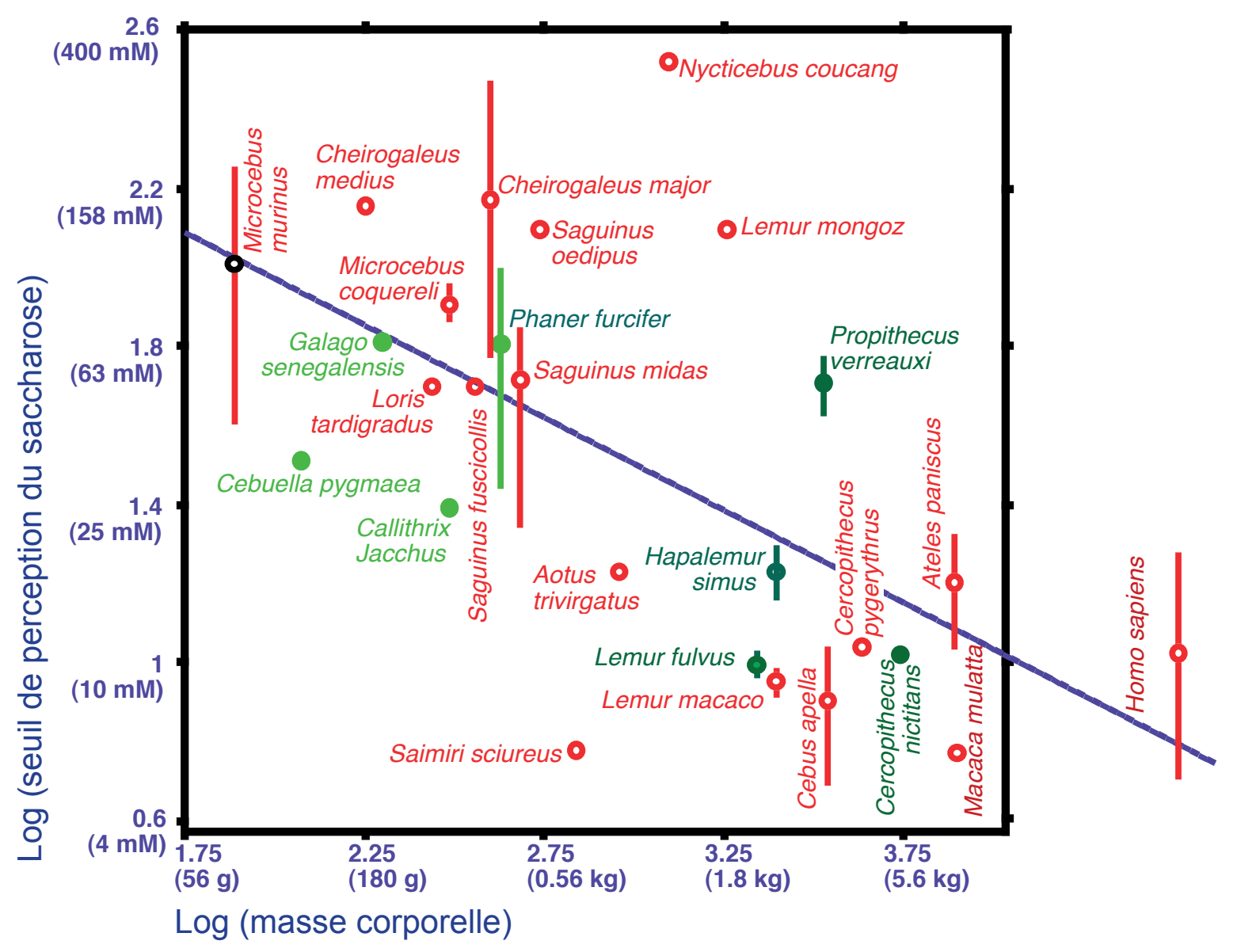

Figure 1.

Relation entre le seuil de perception du saccharose en solution aqueuse (concentrations en millimoles) et le poids corporel de différentes espèces de primates. Relationship between gustatory thresholds for sucrose solutions (concentrations in $\mathrm{mM}$ ) and the body weight of various primate species (including humans). 


\section{L'inertie phylogénétique : le cas de la perception du fructose}

Nous avons regroupé (Fig. 2) nos résultats [4] concernant la mesure de la sensibilité gustative visà-vis du fructose - l'un des sucres solubles les plus fréquents dans les pulpes de fruits d'Angiospermes - chez deux séries d'espèces de primates apparentés, les Lemuridae (lémurs et hapalémurs de Madagascar) et les Callitrichidae (ouistitis et tamarins d'Amérique du sud). On remarque que les seuils de discrimination du fructose tendent vers des valeurs qui se rapprochent chez les espèces appartenant à un même groupe taxonomique en dépit des tailles variables et des tendances alimentaires distinctes au sein de chaque groupe d'espèces - avec des régimes largement constitués de polysaccharides (feuilles, exsudats végétaux) ou faisant une plus large part aux sucres solubles (pulpes des fruits).

\begin{tabular}{|c|c|c|c|c|}
\hline & Taxon & Poids (g) & Fructose $(\mathrm{mM})$ & \\
\hline 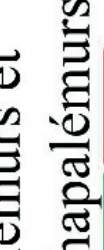 & $\begin{array}{l}\text { Lemuridae } \\
\text { Eulemur coronatus } \\
\text { Eulemur fulvus } \\
\text { Eulemur macaco } \\
\text { Hapalemur simus } \\
\text { Hapalemur griseus }\end{array}$ & $\begin{array}{l}2200 \\
2200 \\
2500 \\
2500 \\
850\end{array}$ & $\begin{array}{l}21 \pm 10 \\
22,5 \pm 8,5 \\
14 \pm 7 \\
18,5 \pm 7,5 \\
16,5 \pm 9,5\end{array}$ & $\begin{array}{l}\text { Frugivore- } \\
\text { nectarivore } \\
\text { Folivore }\end{array}$ \\
\hline 岂 & $\begin{array}{l}\text { Callitrichidae } \\
\begin{array}{|l|}\text { Cebuella pygmaea } \\
\text { Callithrix jacchus } \\
\text { Callithrix geoffroyi } \\
\text { Callithrix argentata } \\
\text { Saguinus midas } \\
\text { Saguinus oedipus } \\
\text { Leontopithecus rosalia } \\
\text { Leontopithecus chrysomelas }\end{array}\end{array}$ & $\begin{array}{l}119 \\
300 \\
300 \\
425 \\
492 \\
547 \\
555 \\
555\end{array}$ & $\begin{array}{l}37 \pm 7 ; 50 \\
29,5 \pm 2,5 \\
41 \pm 10 \\
19,5 \pm 7,5 \\
66 \\
16 \pm 6 \\
19,5 \pm 7,5 \\
21,5 \pm 9,5\end{array}$ & $\begin{array}{l}\text { Gommivore- } \\
\text { insectivore } \\
\text { Frugivore- } \\
\text { nectarivore- } \\
\text { insectivore }\end{array}$ \\
\hline
\end{tabular}

Figure 2.

Comparaison des seuils de perception du fructose en solution dans deux séries d'espèces de primates apparentées en fonction des régimes alimentaires. Comparing taste thresholds for fructose among primate species within different taxonomic groups in relation to species major dietary trends.

\section{Dépense énergétique}

Les mesures en conditions naturelles sont limitées à sept espèces de primates non-humains, dont deux d'entre elles ont été testées par les auteurs [10]. Une population humaine dont l'activité physique est importante (communauté Andine agro-pastorale des Aymara), et servant de référence pour l'homme dans notre étude, a été testée avec la méthode de l'eau doublement marquée [11]. La Figure 3 représente la dépense énergétique totale de ces espèces en fonction de leur masse corporelle après transformation logarithmique. La droite obtenue traduit la relation exponentielle qui lie ces deux variables. 


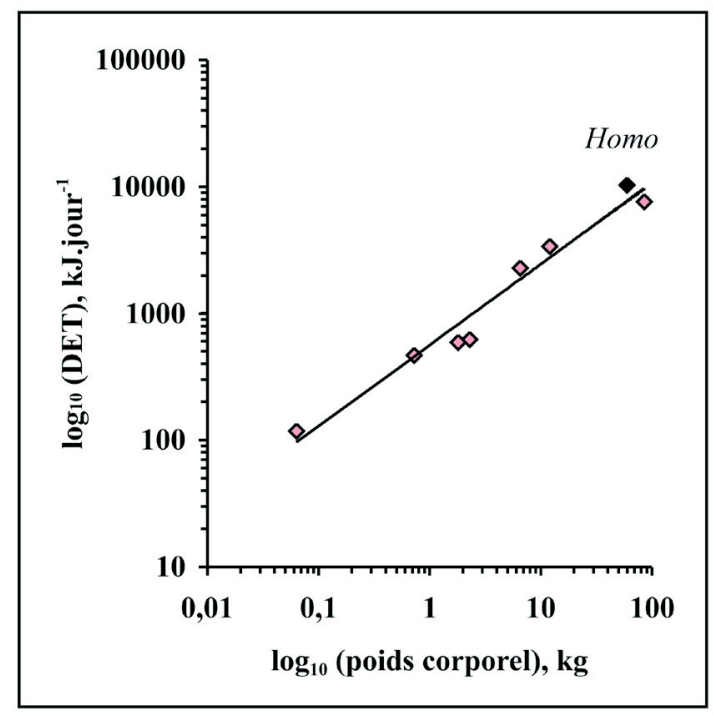

Figure 3.

Relation entre la dépense énergétique journalière (DET, en kJ par jour) et le poids corporel de quelques espèces de primates dont la dépense énergétique a été mesurée, en conditions naturelles, par la méthode à l'eau doublement marquée.

Relationship between daily total energy expenditure (DET, in kJ per day) and body weight of some primate species whose energy expenditure has been measured in natural conditions using the doubly labeled water method.

En fait, la dépense énergétique totale des primates non humains (Figure 4) se situe à un niveau sensiblement inférieur à celle des autres mammifères placentaires, à l'exception du microcèbe dont les mécanismes de conservation de l'énergie sont très particuliers [12]. Selon cette relation qui porte sur des espèces de tailles très distinctes et arborant de styles de vie et des adaptations variables au régime alimentaire, les primates dans leur ensemble pourraient être considérés comme des espèces à mode de vie «économe ».

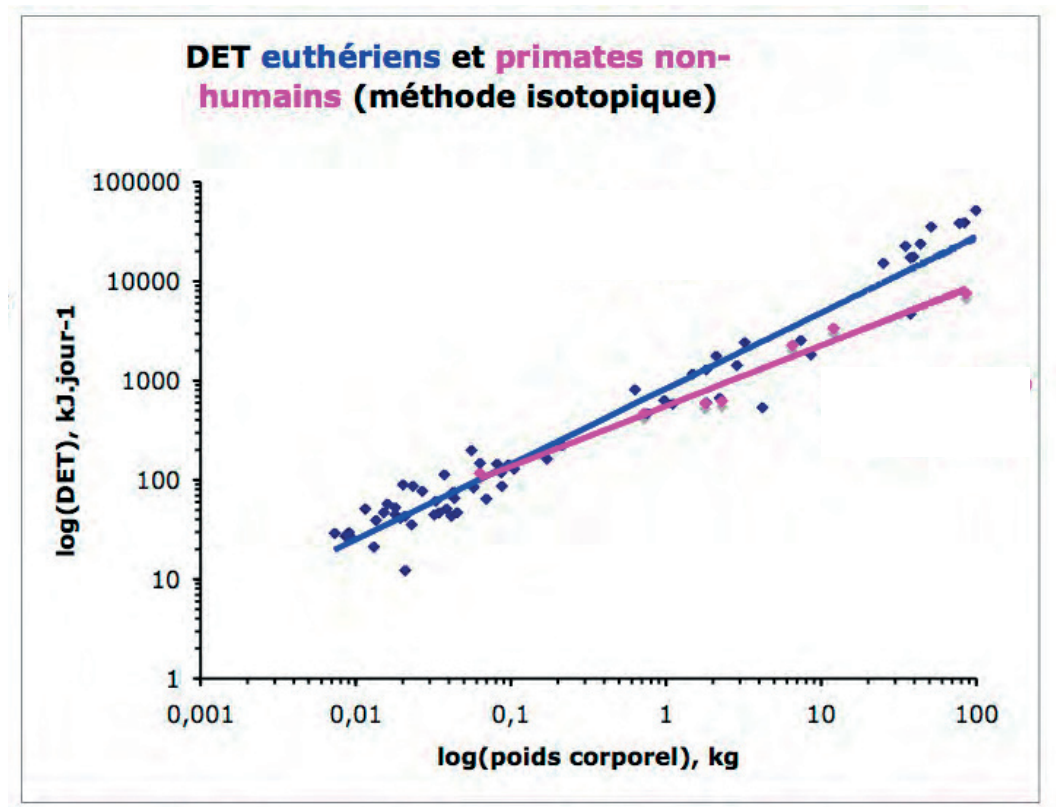

Figure 4.

Relations entre le poids corporel et la dépense énergétique totale journalière (DET, en kJ par jour) chez l'ensemble des mammifères euthériens (hors primates) pour lesquels la méthode isotopique de mesure (à l'eau doublement marquée) a été pratiquée, et chez les primates non humains pour lesquels cette relation allométrique a été analysée séparément.

Relationships between body weight and total daily energy expenditure (DET, in kJ per day using doubly labeled water) among eutherian mammals (except primates), compared to primates for which the allometric relationship was calculated separately. 


\section{Discussion}

Les stratégies alimentaires des Primates non humains et la perception gustative Les besoins énergétiques importants des primates de grande taille sont couverts par une alimentation diversifiée associée à un vaste domaine vital et de grandes distances parcourues quotidiennement en quête de nourriture. Pour ces espèces, le maintien d'un régime éclectique repose en partie sur l'acuité gustative élevée vis-à-vis des substances à haute valeur énergétique, comme les sucres solubles : elle permet en effet d'élargir l'éventail des produits perçus comme palatables et comestibles, y compris les fruits à très faible teneur en sucres qui sont négligés par les petites espèces, moins sensibles au saccharose. Les primates de grande taille ne pourraient pas, en effet, couvrir leurs besoins énergétiques en se spécialisant sur les seules ressources à haute qualité nutritionnelle, très dispersées dans l'environnement $[4,8]$.

Inversement, les primates de petite taille comme le microcèbe (Microcebus murinus), souvent très mobiles, chassent des petites proies animales et consomment les parties reproductrices des végétaux riches en énergie (nectars, fruits mûrs). Bien qu'en valeur absolue leurs besoins énergétiques soient modérés du fait de leur petite taille, ces espèces ont un métabolisme de base par unité de temps beaucoup plus important que les grands primates et la quête d'aliments riches en énergie permet de subvenir à ces besoins. Le seuil élevé de perception gustative (faible sensibilité) chez ces espèces favorise sans doute leurs choix alimentaires vers les éléments plus riches en sucres, les seuls qu'ils perçoivent réellement comme attractifs $[4,8]$.

La signification fonctionnelle des sensibilités gustatives, présentée ici, n'exclut pas cependant de considérer les particularités sensorielles de certaines espèces en fonction de l'inertie phylogénétique. Ainsi, nous interprétons la similitude des seuils de perception, du fructose par exemple chez des espèces proches phylogénétiquement comme les ouistitis et les tamarins, mais de taille distincte, comme une inertie génétique. Elle résulterait de la lenteur des adaptations à des milieux qui peuvent se transformer rapidement (dans le cas, par exemple de la colonisation par de nouvelles espèces de plantes).

Nous connaissons un exemple extrême de possibilité de perception liée à la phylogénie : le cas des «faux sucres ». Ces substances, perçues par l'homme (et les primates de l'Ancien Monde) comme extrêmement sucrées mais dénuées de valeur calorique - par exemple la pulpe du fruit de Pentadiplandra brazzeana, une espèce africaine - , ne sont absolument pas perçues par les primates du Nouveau Monde [13]. En fait il s'agit d'une co-évolution des espèces d'Angiospermes à fruits pulpeux avec les primates qui disséminent les graines dans les forêts denses africaines. Le mimétisme biochimique avec les « vrais » sucres a favorisé les espèces végétales dont le goût des fruits ressemble à celui des nombreuses espèces à fruits sucrés recherchés par les primates. Les «faux sucres » sont généralement des protéines qui, même en très faibles concentrations, présentent un goût extrêmement sucré pour l'homme. Cette caractéristique n'est pas observée chez les primates du Nouveau Monde (qui ne discriminent pas une solution de «faux-sucre » de l'eau pure). Cette dichotomie sensorielle entre les deux grands groupes de primates correspond à une évolution disjointe se traduisant par une différence des protéines des récepteurs gustatifs. La différenciation des systèmes gustatifs entre ces groupes remonte à la séparation des plaques continentales Afrique/Amérique. Elle ne permet pas aux primates du Nouveau Monde de percevoir les « faux sucres », actuellement décrits uniquement pour les plantes de l'Ancien Monde. Ces «faux sucres» sont nécessairement apparus plus tardivement sur le continent Africain en fonction de la perception gustative des espèces alors présentes [13], dont est issue la lignée humaine.

\section{L'homme a-t-il une place à part du point de vue des stratégies énergétiques ?}

Les apports énergétiques doivent nécessairement équilibrer les dépenses énergétiques. Le plus souvent, le métabolisme global - incluant, outre les besoins métaboliques de base, les coûts de l'activité locomotrice et de l'alimentation, les dépenses de thermorégulation et les coûts de toutes les activités liées aux rapports sociaux dans les groupes - , est calculé par la méthode factorielle, rigoureuse et calibrée en ce qui concerne l'humain, mais qui peut accumuler de petites erreurs sur le calcul de chaque type d'activité pour des primates non humains observés en conditions naturelles.

La méthode de l'eau doublement marquée [1,6] appliquée chez les primates non humains en conditions naturelles, et chez l'homme, montre que la dépense énergétique totale varie en fonction de la masse 
corporelle des espèces. Si le groupe des primates dans son ensemble s'avérait présenter un métabolisme global sensiblement réduit par rapport aux autres groupes de mammifères, comme le suggère la plus faible dépense énergétique totale de plusieurs espèces de primates pour une masse corporelle donnée, la notion de «primate économe » pourrait s'appliquer à l'humain. Une plus faible dépense énergétique au cours des différentes activités journalières peut s'expliquer en partie en fonction de la moindre masse musculaire des primates non-humains en comparaison des autres mammifères placentaires de taille similaire [14].

Dans cette hypothèse, l'impact d'une moindre activité physique en termes de prévalence de l'obésité dans les sociétés occidentales [15] ne reflèterait pas seulement des particularités socio-économiques et culturelles. Dans l'éventualité d'une particularité énergétique propre à l'ensemble des primates, notre compréhension des mécanismes de régulation de l'équilibre énergétique et des facteurs d'adiposité dans les populations humaines passées et présentes se trouverait sensiblement modifiée. C'est dans un contexte de grande sensibilité gustative et de couverture des besoins métaboliques accrus par l'augmentation du volume de son encéphale, que le genre Homo s'est différencié des autres primates, en particulier en adaptant son alimentation par la cuisson. Ce traitement culinaire particulier non seulement accroît les apports énergétiques mais procure un plaisir gustatif qui n'est certainement pas étranger au succès (ou aux excès actuels) de cette nouvelle forme d'alimentation.

\section{Références}

1. Tarnaud L, Garcia C, Krief S, Simmen B (2010) Apports nutritionnels, dépense et bilan énergétiques chez l'homme et les primates non-humains : aspects méthodologiques. Rev Primatol 3 http://primatologie. revues.org/558

2. Hladik CM (2011) Le feu de la vie va-t-il consumer la Terre ? In: Hladik J (ed) Les énergies renouvelables aujourd'hui et demain. Ellipses, Paris, pp 103-119

3. Nagy KA, Girard IA, Brown TK (1999) Energetics of free-ranging mammals, reptiles, and birds. Ann Rev Nutr 19:247-277

4. Simmen B, Hladik CM (1998) Sweet and bitter taste discrimination in Primates: scaling effects across species. Folia Primatol 69:129-138

5. Simmen B, Pasquet P, Hladik CM (2004) Methods for assessing tatse abilities and hedonic responses in human and nonhuman primates. In: Macbeth $\mathrm{H}$, MacClancy $\mathrm{J}$ (eds) Researching food habits. Methods and problems. Berghahn Books, New York, pp 87-99

6. Lifson N, McClintock R (1966) Theory of use of turnover rates of body water for measuring energy and material balance. J Theor Biol 12:46-74

7. Glaser D (1986). Geschmacksforschung bei Primaten. Sonderdr Vierteljahrsschr

Naturforsch Ges Zurich 131:92-110

8. Hladik CM, Simmen B (1996) Taste perception and feeding behavior in non-human primates and human populations. Evol Anthropol 5:58-71

9. Simmen B, Hladik CM (1993) Perception gustative et adaptation à l'environnement nutritionnel des Primates non humains et des population humaines. Bull Mém Soc Anthrop Paris, n.s. 5:343-354

10. Simmen B, Bayart F, Rasamimanana H, et al (2010) Total energy expenditure and body composition in two free-living sympatric lemurs. PLOS ONE 5(3):e9860 
11. Kashiwazaki H, Uenishi K, Kobayashi T, et al (2009) Year-round high physical activity levels in agropastoralists of Bolivian Andes: results from repeated measurements of DLW method in peak and slack seasons of agricultural activities. Am J Hum Biol 21:337-45

12. Perret M, Aujard F, Vannier G (1998) Influence of daylength on metabolic rate and daily water loss in the male prosimian primate Microcebus murinus. Comp Biochem Phys A

119:981-989

13. Pasquet P, Hladik CM, Tarnaud L (2011) Evolution des perceptions gustatives. Biofutur 320:38-42

14. Muchlinski MN, Snodgrass JJ, Terranova CJ (2012) Muscle mass scaling in primates: an energetic and ecological perspective. Am J Primatol 74:395-407

15. Hayes M, Chutsek M, Heska S et al. (2005) Low physical activity levels of modern Homo sapiens among free-ranging mammals. Int J Obes 29:151-156 